\title{
LUNA results on deuterium burning and implications for cosmology
}

\author{
Federico Ferraro ${ }^{a, 1, *}$ \\ ${ }^{a}$ Università degli Studi di Milano and INFN, Sezione di Milano, \\ Via Celoria 16, 20133 Milan, Italy \\ E-mail: federico.ferraro@mi.infn.it
}

Lightest elements were produced in the first few minutes of the Universe through a sequence of nuclear reactions known as Big Bang nucleosynthesis (BBN). Although astronomical observations of primordial deuterium abundance have reached percent accuracy, theoretical predictions based on BBN are affected by the large uncertainty on the cross-section of the $\mathrm{D}(\mathrm{p}, \gamma)^{3} \mathrm{He}$ deuterium burning reaction. Here I am reporting on a new measurement of the $\mathrm{D}(\mathrm{p}, \gamma)^{3} \mathrm{He}$ cross section performed by the LUNA collaboration to an unprecedented precision of better than $3 \%$. This result settles the most uncertain nuclear physics input to BBN calculations and substantially improve the use of primordial abundances as probes of the physics of the early Universe.

\footnotetext{
*** Particles and Nuclei International Conference - PANIC2021 ***

*** 5 - 10 September, 2021 ***

$* * *$ Online $* * *$

${ }^{1}$ on behalf of the LUNA collaboration

* Speaker
} 


\section{Introduction}

The combination of different results on the observed Deuterium abundance relative to Hydrogen in low metallicity systems recently provided a high-precision measurement of this quantity, yielding $(\mathrm{D} / \mathrm{H})_{\mathrm{obs}}=(2.527 \pm 0.030) \times 10^{-5}[1]$. On the other hand, Big Bang Nucleosynthesis $(\mathrm{BBN})$, combined with the $\Lambda$-Cold Dark Matter $(\Lambda \mathrm{CDM})$ model and the high-precision measurements of the Cosmic Microwave Background ( $\mathrm{CMB}$ ), provides an estimation of the primordial Deuterium abundance: $(\mathrm{D} / \mathrm{H})_{\mathrm{BBN}}$. The estimation, though, was not yet as precise as the measured value, mainly because of the uncertainty on the nuclear physics input to the BBN reaction network. In particular, the $\mathrm{D}(\mathrm{p}, \gamma)^{3} \mathrm{He}$ reaction was the most uncertain in the network and different values of $(\mathrm{D} / \mathrm{H})_{\mathrm{BBN}}$ have been obtained in the past $[2,3]$, advocating the need of new, precise direct measurements of the cross section in the energy range relevant to BBN $\left(E_{c m} \approx 30-300 \mathrm{keV}\right)$. In fact, while the cross section of the $\mathrm{D}(\mathrm{p}, \gamma)^{3} \mathrm{He}$ reaction was already well known at low energies $\left(E_{c m} \approx 3-20 \mathrm{keV}\right)$, systematic uncertainties of 8-9\% affected the cross section at energies relevant to BBN. The primordial abundances of light elements are strongly influenced by the baryon-tophoton ratio $\eta$, which is closely related to the baryon density $\Omega_{b}$, so, under a certain hypothesis on the effective number of neutrino species $N_{\text {eff }}$, the comparison of $(\mathrm{D} / \mathrm{H})_{\mathrm{obs}}$ and $(\mathrm{D} / \mathrm{H})_{\mathrm{BBN}}$ lead to the determination of $\Omega_{b}$.

A new measurement of the $\mathrm{D}(\mathrm{p}, \gamma)^{3} \mathrm{He}$ reaction cross section has been recently performed [4] at the Laboratory for Underground Nuclear Astrophysics (LUNA) $400 \mathrm{kV}$ accelerator [5], in the Gran Sasso National Laboratory (LNGS), in Italy.

\section{Experiment}

Thanks to the $1400 \mathrm{~m}$ thick rock overburden, LUNA can benefit from a million-fold reduction in the flux of muons caused by the interaction of cosmic rays in the atmosphere [6]. As a result, the only significant contribution to the laboratory $\gamma$ background is due to natural radioactivity of the surrounding materials and rocks, which becomes negligible above the ${ }^{208} \mathrm{Tl}$ line at $E_{\gamma}=2614.5 \mathrm{keV}$. For this reason, LUNA is particularly suited for the direct measurement of nuclear cross sections in the energy interval of astrophysical or cosmological interest [7], often characterized by low counting rate and $\gamma$ rays above the ${ }^{208} \mathrm{Tl}$ line. Some beam-induced background was present above $E_{p}=250 \mathrm{keV}$ though, mainly due to the ${ }^{19} \mathrm{~F}(\mathrm{p}, \alpha \gamma){ }^{16} \mathrm{O}$ reaction and its Compton continuum. Runs with Helium inside the target chamber were taken to properly subtract the beam-induced background.

The present measurement was performed thanks to the $400 \mathrm{kV}$ electrostatic accelerator, able to deliver a $50-400 \mathrm{keV}$ proton beam as intense as $500 \mu \mathrm{A}$, with very high long-term stability that enable long-lasting measurements. The windowless, differential-pumping, extended gas target (see figure 1) was used to ensure both high target purity and low beam energy degradation. The target consisted of deuterium gas at 0.3 mbar contained in a $33 \mathrm{~cm}$ long interaction chamber, continuously flushed from a bottle of $99.999 \%$ pure deuterium through the pressure-control system that kept the pressure stable at the $0.25 \%$ level [8]. To know the density profile and the number of target nuclei along the beam path, pressure and temperature were measured in several points by means of capacitance pressure gauges and Pt100 resistive temperature detectors respectively. The previous 


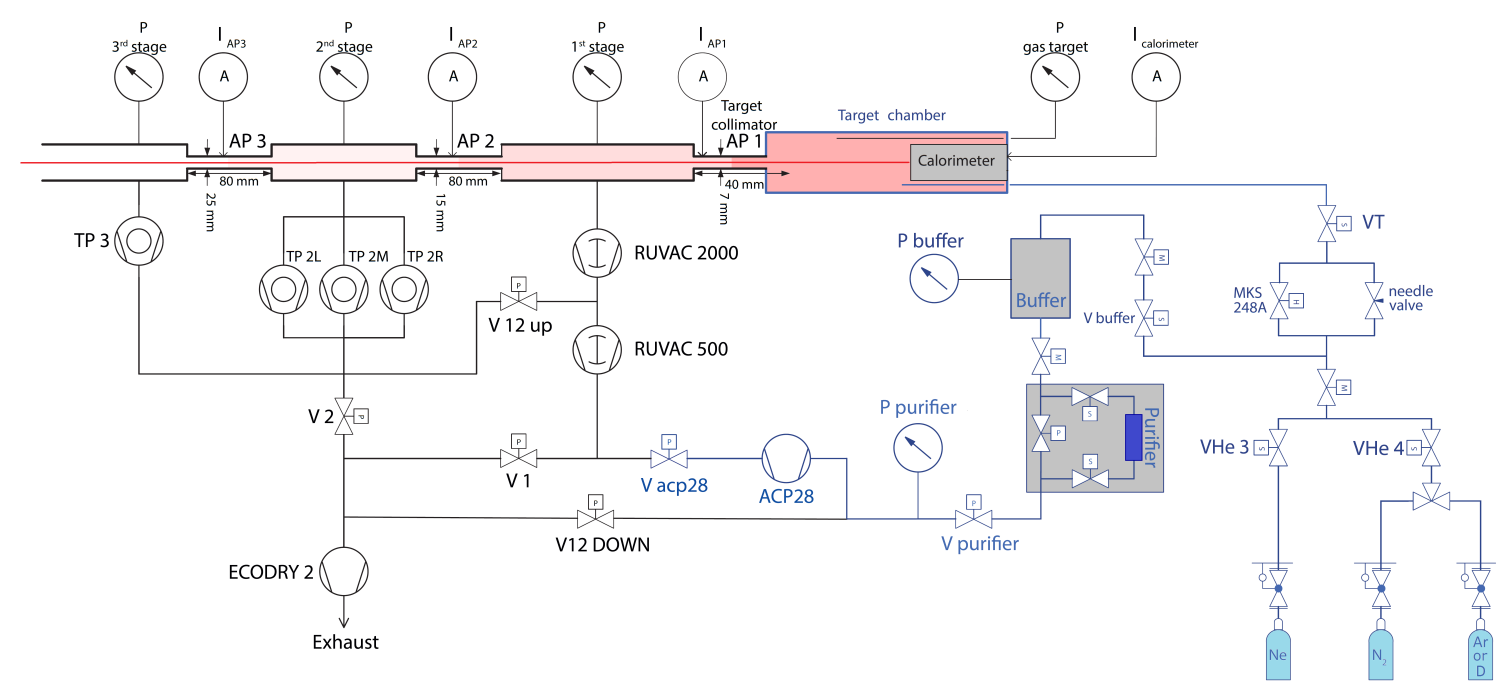

Figure 1: LUNA windowless, differential-pumping, extended gas target. Reddish color represents the gas pressure: the more intense, the higher the gas pressure. The red line represents the proton beam. High-purity gas lines are represented in blue.

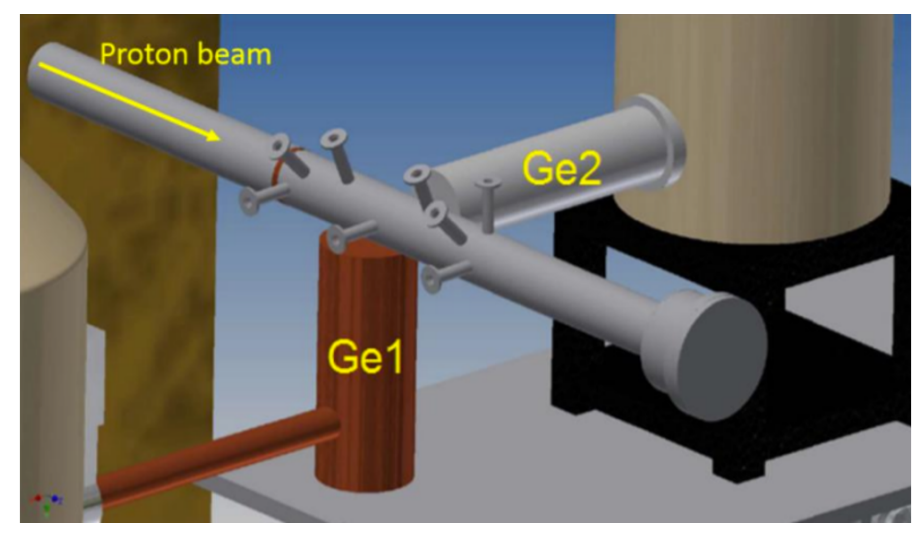

Figure 2: Sketch of the interaction chamber with side tubes for pressure and temperature measurements and the two HPGe detectors used in the experiment.

calorimetric system used to measure the beam current [9] was upgraded to meet the precision and accuracy requirements for the present measurement, set to $1 \%$.

A large, High-Purity Germanium (HPGe) detector in close geometry (Ge1 in figure 2) was used to detect $\gamma$ rays from the $\mathrm{D}(\mathrm{p}, \gamma)^{3} \mathrm{He}$ reaction with high resolution and reasonably high efficiency. The detection efficiency was measured in several points along the beam path by means of a second HPGe detector (Ge2 in figure 2), using the coincidence method. The efficiency measurements were then used to fine-tune a Monte Carlo simulation that enables a precise evaluation of the detection efficiency as a function of the position of the interaction and the photon energy, which depends on the emission angle.

The $\mathrm{D}(\mathrm{p}, \gamma)^{3} \mathrm{He}$ reaction has a $Q$-value of $5.493 \mathrm{MeV}$. The proton is captured in the ground state of ${ }^{3} \mathrm{He}$ and a $\gamma$ ray is emitted with energy $E_{\text {gamma }}=Q+E_{c m}$, where $E_{c m}$ is the proton energy in the center of mass reference frame. The reaction, though, may take place in different positions 
along the beam path, both upstream and downstream w.r.t. the detector, and the Doppler effect causes a broadening of the full energy peak in the $\gamma$ spectrum, hence the observed shape of the full energy peak is related to the $\gamma$ angular distribution. The observed angular distribution, together with the precise measurement of the cross section (hence the astrophysical factor $S(E)$ ) allows the validation of ab-initio nuclear models [10].

\section{Discussion}

LUNA measured the cross section of the $\mathrm{D}(\mathrm{p}, \gamma)^{3} \mathrm{He}$ reaction at 12 different energies between $E_{c m}=33 \mathrm{keV}$ and $263 \mathrm{keV}$. The derived $S$-factor lies between the best fit of previous experimental data [11] and the theoretical calculation within the ab-initio model [10]. The new fit including all experimental data is completely dominated by the new results by LUNA, being the uncertainty drastically reduced w.r.t. previous measurements.

Considering an effective number of neutrinos $N_{e f f}=3.045$, the numerical BBN code PArthENoPE [12] was used to calculate the baryon density $\Omega_{b}$ [4]. The result is consistent with Planck and is more accurate and precise than previous results based on preceding measurements of the $\mathrm{D}(\mathrm{p}, \gamma)^{3} \mathrm{He}$ cross section.

To further probe the dark sector and the existence of physics beyond the $\Lambda \mathrm{CDM}$ model, a second analysis was performed, letting the effective number of neutrinos $N_{\text {eff }}$ free to vary. Two slightly different likelyhood analysis, implementing different priors on the baryon density, determined a value of $N_{e f f}$ consistent with 3, giving no evidence for the existence of dark radiation.

\section{References}

[1] R.J. Cooke, M. Pettini and C.C. Steidel, One percent determination of the primordial deuterium abundance, The Astrophysical Journal 855 (2018) 102.

[2] A. Coc, P. Petitjean, J.-P. Uzan, E. Vangioni, P. Descouvemont, C. Iliadis et al., New reaction rates for improved primordial $\mathrm{D} / \mathrm{H}$ calculation and the cosmic evolution of deuterium, Phys. Rev. D 92 (2015) 123526.

[3] C. Pitrou, A. Coc, J.-P. Uzan and E. Vangioni, Precision big bang nucleosynthesis with improved helium-4 predictions, Physics Reports 754 (2018) 1.

[4] V. Mossa, K. Stöckel, F. Cavanna, F. Ferraro, M. Aliotta, F. Barile et al., The baryon density of the universe from an improved rate of deuterium burning, .

[5] A. Formicola, G. Imbriani, M. Junker, D. Bemmerer, R. Bonetti, C. Broggini et al., The luna ii 400kv accelerator, Nuclear Instruments and Methods in Physics Research Section A: Accelerators, Spectrometers, Detectors and Associated Equipment 507 (2003) 609.

[6] C. Broggini, D. Bemmerer, A. Caciolli and D. Trezzi, LUNA: Status and Prospects, Prog. Part. Nucl. Phys. 98 (2018) 55. 
[7] F. Cavanna and P. Prati, Direct measurement of nuclear cross-section of astrophysical interest: Results and perspectives, International Journal of Modern Physics A 33 (2018) 1843010.

[8] V. Mossa, K. Stöckel, F. Cavanna, F. Ferraro, M. Aliotta, F. Barile et al., Setup commissioning for an improved measurement of the $\mathrm{D}(\mathrm{p}, \gamma)^{3} \mathrm{He}$ cross section at big bang nucleosynthesis energies, The European Physical Journal A 56 (2020) 144.

[9] F. Ferraro, M.P. Takács, D. Piatti, V. Mossa, M. Aliotta, D. Bemmerer et al., A high-efficiency gas target setup for underground experiments, and redetermination of the branching ratio of the $189.5 \mathrm{kev} 22 \mathrm{ne}(p, \gamma) 23 \mathrm{na}$ resonance, .

[10] L.E. Marcucci, G. Mangano, A. Kievsky and M. Viviani, Implication of the proton-deuteron radiative capture for big bang nucleosynthesis, Phys. Rev. Lett. 116 (2016) 102501.

[11] E.G. Adelberger, A. García, R.G.H. Robertson, K.A. Snover, A.B. Balantekin, K. Heeger et al., Solar fusion cross sections. ii. the pp chain and cno cycles, Rev. Mod. Phys. 83 (2011) 195.

[12] R. Consiglio, P. de Salas, G. Mangano, G. Miele, S. Pastor and O. Pisanti, Parthenope reloaded, Computer Physics Communications 233 (2018) 237. 